\title{
SIMULATION OF LARGE AGGREGATE PARTICLES SYSTEM WITH A NEW MORPHOLOGICAL MODEL
}

\author{
Giulia Ferri $^{1}$, Severine Humbert ${ }^{1}$, Mathieu Digne ${ }^{1}$, Jean-Marc Schweitzer ${ }^{1}$ \\ AND MAXIME MOREAUD ${ }^{\bowtie, 1,2}$ \\ ${ }^{1}$ IFP Energies Nouvelles, Rond-Point de l'échangeur de Solaize; 69360, Solaize, France, ${ }^{2}$ MINES ParisTech, \\ PSL-Research University, CMM, 77305 Fontainebleau, France \\ e-mail: ferrigiulia943@gmail.com, severine.humbert@ifpen.fr, mathieu.digne@ifpen.fr, \\ jean-marc.scwehtzer@ifpen.fr,maxime.moreaud@ifpen.fr \\ (Received December 1, 2020; revised April 23, 2021; accepted May 19, 2021)
}

\begin{abstract}
For the development of a new porous material such as catalytic carrier, the control of the textural properties is of fundamental importance. In order to move towards rational synthesis, it is necessary to better understand the physical phenomena that generate a defined solid structure. A contribute to this purpose can be achieved by studying the aggregation process inside colloidal suspensions, leading to porosity generation: this phenomenon can be described with a Brownian dynamics model that, for any set of chemical parameters, gives access to the mass distribution and the fractal dimension of colloidal aggregates. However, this model cannot be used for the simulation of large colloidal systems, due to its high computational time, limiting comparison with analytical methods, which probe the whole multi-scale system. This problem is solved by developing a new aggregation morphological model, wherein the fractal dimension is tuned with two compactness parameters. An efficient simulation algorithm is proposed in case of spheres, for which the fractal dimension of the generated aggregates varies between 1.2 and 3. Brownian dynamics results are used to parametrize this purely geometric model, in order to constrain the size and the morphology of the aggregates created. The large numerical solid will be representative of the textural properties of a real solid and will give more information on the porous network. It could be used, for example, to simulate diffusive transport coupled with chemical reaction and to study the impact of the geometry of the porous system on the catalytic performance.
\end{abstract}

Keywords: Colloidal structure, Fractal dimension, Morphological model.

\section{INTRODUCTION}

In catalytic carriers and absorbents field, the control of textural properties is of fundamental importance. The void fraction, the pore size distribution and the pore's interconnection affect the mass and heat transport inside the solid structure. The textural properties are the result of the preparation process: most of meso- and/or macroporous of industrial interest, such as silica (Iler, 2004) and alumina (Euzen, 2002), exhibit a multi-scale porosity based on particles stacking. For instance, a $\gamma$-alumina support consists of elementary crystallites (often nanometric platelets (Chiche, 2008), whose size and shape result from the alumina precursors, such as boehmite $\mathrm{AlOOH}$ ). These crystallites aggregate to form aggregates of a few tens of nanometers generating mesoporosity. Further, these aggregates can then agglomerate to form agglomerates of a few microns, generating macroporosity. This complex assembly process is not random and results from the inter-particles interactions during the different preparation steps. This interactions can be largely tuned: for instance, $\mathrm{pH}$, ionic strength and concentration have strong impact on the solid formed during the agglomeration process of a boehmite suspension in Brownian conditions (Anovitz, 2018). Such suspension agglomeration is encountered for instance during the boehmite synthesis or the shaping process. There is also a need to increase competences and knowledge of the fundamental mechanisms involved in the aggregation processes.

The effect of the synthesis conditions on the final dry solid multi-scale structure can be evaluated experimentally, by several techniques like Small Angle X-Ray Scattering (SAXS) (Sasanuma, 1989) and Nitrogen physisorption (Munhoz, 2014). An alternative way is to use numerical simulations, indeed, the description of the agglomeration kinetics with physical models, such as DLVO (Dergajuin, Landau, Verwey and Overbeek) (Verwey, 1948) theory enables to estimate the size distribution and fractal dimension of colloidal agglomerates for any set of $\mathrm{pH}$, ionic strength and concentration. Nevertheless, a realistically large porous material cannot be generated on the sole basis of DLVO dynamics modeling, due to high computational cost simulating large particle systems.

In order to solve this problem, we propose a 
new morphological aggregation model, able to build aggregates of variable fractal dimension, generalized to any geometry of elementary particles.

Lagrangian models provide reference systems to parameterize the morphological aggregation model, where the interaction potential amongst particles is described by the DLVO theory (Verwey, 1948) and Brownian motion is included using Langevin force (Langevin, 1908). The mass distribution and the aggregate fractal dimension extracted from the final arrangement of aggregates allow the parametrization of the morphological aggregation model. By adjusting two compactness parameters and the number of elementary object contained in each cluster, this model is able to build the same aggregates obtained with Lagrangian simulations.

This paper presents the new morphological aggregation model and the strategy for its parametrization. In the following paragraph we present the equations used in the Lagrangian model. The result of such a model constitutes our reference system. From the final arrangement it is possible to determine the distribution of structural parameters (number of particles constituting the aggregates, fractal dimension) necessary for a realistic description of colloidal systems. In the following part, after a brief review of the different families of packing models, we explain the formalism of the morphological aggregation model, with a focus on the role of the compactness parameters. An acceleration of the process is also proposed for the case of spheres with constant radius. For this case, the strategy for the parametrization of the aggregation model using the Lagrangian model is presented. Finally, the relationship between the structural parameters of the colloidal system and the textural properties of the final porous solid is exemplified. The natural perspective of this work is to simulate colloidal aggregates to build a numerical solid, and is addressed in the discussions section. Conclusions are drawn in the end.

\section{CONTRIBUTION OF BROWNIAN- DLVO MODEL}

\section{MODELS AND THEORY}

The numerical simulation of particle dynamics is of great interest in the material science research community (Lu, 2008)(Krinninger, 2016), due to the possibility of studying the various properties of soft matter systems such as gels (Zaccarelli, 2007), polymers (Nikunen, 2007) and suspensions (Cerbelaud, 2010). These purely physical methods strongly integrate or may be an alternative to experimental approaches in exploring the timedependent behaviour of silica (Lebdioua, 2020) and alumina (Laganapan, 2015) colloidal systems, giving micro-structural information (e.g. fractal dimension, porosity (Hutter, 2000)) that is not always accessible experimentally, for different sets of chemicalphysical parameters $(\mathrm{pH}$, ionic strength, concentration (Cerbelaud, 2010)) and flow conditions. Amongst the different approaches, Brownian Dynamics is largely applied due to its relatively simple implementation and capability of being easily generalized (Park, 2016). The main drawbacks are that the computational effort scales unfavorably for large systems (Dugosz, 2011).

The boehmite aggregation process is simulated with Brownian Dynamics coupled with the DLVO theory. The model calculates the dynamics of identical spherical particles by solving the momentum balance

$$
m_{i} \cdot \vec{a}_{i}=\sum_{i \neq j} \vec{F}_{i j}+\vec{F}_{i}^{R}+\vec{F}_{i}^{D},
$$

where $m_{i}$ and $a_{i}$ are the mass and the acceleration of the particle $i, \vec{F}_{i j}$ is the interaction force between $i$ and the particles $j$, deriving from DLVO potential, $\vec{F}_{i}^{R}$ is the random Langevin force, which represents the effect of random collisions with solvent molecules causing Brownian motion, $\overrightarrow{F_{i}^{D}}$ is the viscous drag force acting on $i$.

The DLVO potential $U_{D L V O}$ for two identical spheres is the sum of an attractive potential $U_{v d W}$ (van der Waals potential) and a repulsive potential $U_{e l}$ (electric potential). These potentials can be calculated for identical spheres ((Hamaker, 1937), (Verwey, 1948)) as

$$
\begin{gathered}
U_{v d W}=-\frac{A_{H}}{6}\left(\frac{2 a^{2}}{r^{2}-4 a^{2}}+\frac{2 a^{2}}{r^{2}}+\ln \left(\frac{r^{2}-4 a^{2}}{r^{2}}\right)\right) \\
U_{e l}=\frac{64 \pi k_{B} T n_{\text {ion }} a}{K_{\text {debye }}^{2}} \gamma_{G}^{2} e^{-K_{\text {debye }} h}
\end{gathered}
$$

with

$$
\begin{aligned}
& h=r-2 a \\
& \gamma_{G}=\tanh \left(\frac{e \psi_{o}}{4 k_{B} T}\right) \\
& K_{\text {debye }}=\left(\frac{\varepsilon k_{B} T}{2 e^{2} A_{v}}\right)^{-0.5} \sqrt{I}
\end{aligned}
$$

where $A_{H}$ is the Hamaker constant, $a$ is the sphere radius, $r$ is the distance between the centers of the spheres, $k_{B}$ is the Boltzmann constant, $T$ is the temperature, $n_{i o n}$ is the ions number concentration, $e$ is the electron charge, $\psi_{o}$ is the electric surface potential, $\varepsilon$ is the solvent electric permittivity, $A_{v}$ is the Avogadro 
number and $I$ is the ionic strength.

The interaction force between two particles $i$ and $j$ is given by the derivative of the potential (Verwey, 1948)

$$
\vec{F}_{i j}=-\nabla U_{D L V O}
$$

The random Langevin force (Langevin, 1908) is described by

$$
\vec{F}_{i}^{R}=\sqrt{2 k_{B} T k} \vec{W}
$$

where $\vec{W}$ is a random vector whose components are independent Gaussian random numbers with zero mean and unit variance.

The viscous drag force is given by the Stockes law

$$
\vec{F}_{i}^{D}=-6 \pi \mu a
$$

where $\mu$ is the viscosity of the medium. Once the positions of all the spheres are defined, the fractal dimension $d_{f}$ can be calculated with the mass-size relationship (Gmachowski, 2003)

$$
d_{f}=\frac{\log \left(\frac{N}{k f}\right)}{\log \left(\frac{R_{g y r}}{a}\right)},
$$

where $N$ is the number of elementary objects that constitute the aggregate, $R_{g y r}$ is the aggregate radius of gyration and $k_{f}$ is the pre-factor for the fractal scaling relationship whose value is typically between 1 and 2 (Sorensen, 1997).

The geometrical gyration radius $R_{g y r}$ is defined as the mean square of the distances between the spheres' centers and the geometrical center of mass of the aggregate. A more physical definition (Filippov, 2000) contains also $a$, so that in the limit $N=1, R_{\text {gyr }}=a$ :

$$
\begin{aligned}
R_{g y r}^{2}=\frac{1}{N} \sum_{i=1}^{N}\left[\left(x_{i}-x_{g}\right)^{2}\right. & +\left(y_{i}-y_{g}\right)^{2}+ \\
& \left.+\left(z_{i}-z_{g}\right)^{2}+a^{2}\right] .
\end{aligned}
$$

$x_{i}, y_{i}$ and $z_{i}$ are the coordinates of the spheres' centers and $x_{g}, y_{g}$ and $z_{g}$ define the position of the center of mass composed by $N$ identical spheres:

$$
\begin{aligned}
& x_{g}=\frac{\sum_{i}^{N} x_{i}}{N} ; \\
& y_{g}=\frac{\sum_{i}^{N} y_{i}}{N} ; \\
& z_{g}=\frac{\sum_{i}^{N} z_{i}}{N}
\end{aligned}
$$

\section{AGGREGATED REFERENCE SYSTEM}

The objective of this work is to develop a purely geometric model able to mimic the aggregates arrangement obtained with a Brownian Dynamics simulation. As a consequence, we started by generating a reference physical agglomerated system: Fig. 1 shows the Brownian Dynamics final arrangement of 2048 spheres. We used a surface potential $\psi_{o}$ of 1 $\mathrm{mV}$, a ionic strength $I$ of $0.005 \mathrm{M}$ and a solid fraction of $1 \% .27$ days of calculation with a $2.60 \mathrm{GHz}$ CPU are necessary to simulate a time period of $0.278 \mathrm{~s}$, with an integration time step of $3 \cdot 10^{-9} \mathrm{~s}$. The laps time of the physical process are of the order of $10^{-1} \mathrm{~s}$.

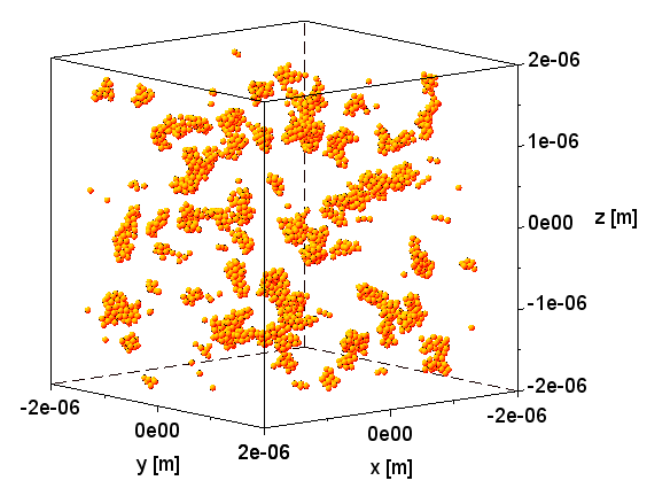

Fig. 1. Final cluster arrangement of a BD DLVO simulation. 2048 identical spheres of radius $40 \mathrm{~nm}$ are initially randomly placed in a cube of length 3800 $\mathrm{nm}$. The interaction potential is calculated with a ionic strength of $0.005 \mathrm{M}$ and a zeta potential of $1 \mathrm{mV}$. The final simulation time is $0.278 \mathrm{~s}$.

If the positions of all elementary spheres are known, it is possible to define the aggregates and for each of them to calculate the mass $(N)$ and the fractal dimension $\left(d_{f}\right)$ using Eq.(7) with $k_{f}=1.2$. Fig. 2 shows the histogram of the mass distribution and the fractal dimensions of each aggregate. Isolated spheres are not taken into account. We can notice that, in number, most of the aggregates have a mass $N$ below 20. In this region, it can be observed that the fractal dimension has a high variability. We can thus deduce from this reference aggregated system that the morphological model should be able to generate fractal aggregates, constituted by a number of particles varying between 1 to almost 150 , and with fractal dimension in the range of 1.2 - 3. From a more global point of view, this model must be able to tune easily the number of elementary particles, their size and shape in order to deal with a wide variety of systems (silica or boehmite for example), but also to allow controlling the fractal dimension within a large range. 


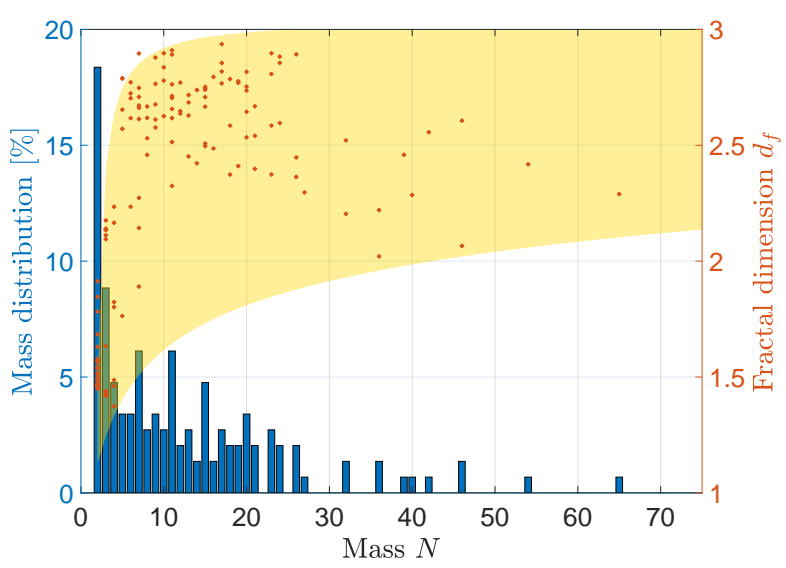

Fig. 2. Mass $(N)$ distribution histogram and fractal dimension $\left(d_{f}\right.$, in red) for the aggregates arrangement obtained with the Brownian dynamics. The yellow area refers to the fractal dimension range of values that can be obtained via the morphological model.

\section{A NEW MORPHOLOGICAL MODEL FOR BUILDING FRACTAL STRUCTURES}

In order to represent the final arrangement of a Brownian Dynamics-DLVO simulation on a larger system, we use a model that takes structural information of fractal dimension $\left(d_{f}\right)$ and number of elementary objects $(N)$ to build aggregates. The modeling of fractal aggregates is a key step for the prediction of the physical properties that depend on their structure, coupled with the experimental analysis of systems of aggregates (Tence, 1986) (Köylü, 1995). The algorithms for the generation of fractal aggregates can be classified in two categories: deterministic models and statistical models. The deterministic, or regular, fractals are generated using an iterative procedure that replaces structural units with smallscale duplicates of the whole cluster Schmidt (1991). This type of fractals is rarely observed in nature and is not representative of real systems, where there is a high degree of disorder. Stochastic algorithms introduce indeed a certain type of randomisation, and can be classified according to the aggregation mechanism, which can be either particle-cluster (Witten, 1983) or cluster-cluster (Meakin, 1983). All the growth mechanisms of intermediate clusters between these two approaches can be divided into classes, associated with a limited range of fractal dimension (Schaefer, 1990). The algorithm we need must be able to access the widest possible range of fractal dimension (ideally between 1 and 3), while the mentioned models are restricted to a reduced range of generated fractal dimension and, in most cases, by a long computational time. Another constraint related to the direct application of these algorithms for the representation of real systems arises from the polydispersity present in nature, and it is therefore necessary to introduce a corresponding size distribution. This problem is considered in several research works (Sorensen, 2001)(Schmidt, 1986). The polydispersity of primary particles is, for example, included in a cluster-cluster algorithm (Tomchuk, 2020) which is an extension of previous algorithms (Touy, 1996) operating according to a hierarchical sequential addition, enabling to obtain precisely a fractal dimension, but requiring high computational effort for the evaluation of all possible sticking positions at each iteration.

For our study, it is necessary to dispose of a model capable of mimicking the aggregates obtained from Lagrangian simulation. Such a model must meet specific criteria: it must perform a sequential addition of grains without overlapping, it must be generalizable to any grain geometry, and the compactness must be controlled. Indeed, the need is to build aggregates starting from elementary objects of any shape, so that we could better represent the multi-scale structure of the porous alumina. In addition, the model must build large systems of aggregates to represent the textural properties of a real solid. To address these issues our approach uses morphological operations to define the sticking positions of a particle on a cluster, thus reducing the computational effort with respect to classical algorithms. Therefore, in our random morphological packing model it is possible to modify the number of elementary objects $(N)$ and compactness, enabling to tune the fractal dimension.

\section{RELATED WORKS}

Packing models can be classified within several families, according to the modeling approach of the physics of contact between objects or according to the order in which the objects fill the space. First, only models with objects of the same size are considered. Random Sequential Addition processes (RSA) (Widom, 1996), allow for a sequential placement of objects in random positions. An object is implanted only if it does not come into contact with any other object already present. This type of simulation is well suited for low-density modeling with low computational time. In variants of this model, already implanted object can be removed to make room for the current object (Matern, 2013). Intrinsic overlapping morphological models can be used for non-overlapping modelling as the dead leaves model (Jeulin, 2000) if only the non-overlapping objects are maintained at the end of the simulation. 
This model produce low-density packings. If low density models are relatively trivial to simulate, this is no longer the case when a high density of objects is desired. Concerning dense simulations, some models (Delarue, 2001) start from an optimal dense arrangement removing randomly some objects to create holes, then add perturbations on objects close to the voids, taking into account collisions. (Lubachevsky, 1990) follows a sequential placement as for the RSA models, and when a new object touches a previously implanted one, the other objects are modified via collisions. It is also possible to use a first non-dense RSA-type model, which is then compressed into a smaller volume (Baranau, 2014), or completed with a non-random placement of spheres (Zhang, 2013). These families of models also require calculations of collisions between objects. Although the number of possible interactions remain limited, the calculation time can quickly become important. A last family of models, with a higher computational time, simulates the filling of a volume through collision during object fall (Seyed-Ahmadi, 2019). They allow extremely realistic simulations, by simulating objects falling into the volume and gradually filling it.

Models with objects of variable size are now considered. The same described approaches can be used. There exist models based on the union of spheres and force-biased approach from molecular dynamics (Moscinski, 1989) for creating packings of fibers (Altendorf, 2011) or any other objects (Donev, 2005). For models with a wide size distribution of objects, with a high ratio between small and large objects size, it is possible to simulate dense packings even with RSA processes (Adamczyk, 1997), or dead leaves models (Jeulin, 2019). The reduction of the number of collision tests via a suitable computational method is often limited to spherical objects (Torquato, 2010). Most of these models define the object locations using real coordinates. However, collision calculations can be simplified with discretizations (Zhang, 2013). There are very few complete approaches based on approximations on discrete grids. It should also be noted that most of the publications concern spherical objects, sometimes ellipsoids.

Our contribution is a new aggregation model, which can be classified within the family of sequential additions, with the originality of proposing a calculation of collisions using a formalism on discrete grids and mathematical morphology. The model is generalized to all geometries of elementary objects. The size of the aggregates formed will depend on the number of elementary objects and on the fractal dimension, the fractal dimension will be tuned by means of two compactness parameters.

\section{AGGREGATION MORPHOLOGICAL MODELS WITH VARIABLE FRACTAL DIMENSION}

A dense simulation model without object overlap is proposed. The objects are either in contact or at a controlled repulsion distance. Using the locations of the implanted objects, the simulation calculates the possible positions for the next object. This model is completed by the definition of concave zones (see Eq.(18)), that further densify the packing.

The probability that the next object is positioned on a concave zone depends on a parameter called $\alpha$ and the probability that the concave zone is the closest to the center of mass of the aggregate depends on a $\beta$ parameter (where $\beta \leq \alpha$ ) (Fig.3).

A fast calculation method will be proposed in the case of simulation with spheres of constant radius on a discrete grid.

Basic morphological operations on sets are recalled. Considering $X$ defined by a set of points $z$ in $\mathbb{R}^{3}$, its complementary set is written as $X^{c}=$ $\left\{z \in \mathbb{R}^{3}, z \notin X\right\}$. The position of $X$ is denoted as the position of one of its points in $\mathbb{R}^{3}$. The morphological dilation $\delta_{X}(Y)$ and erosion $\varepsilon_{X}(Y)$ of the set $\mathrm{Y}$ by $\mathrm{X}$ are respectively Serra (1988):

$$
\begin{aligned}
& \delta_{X}(Y)=\left\{z: \breve{X}_{z} \cap Y \neq \emptyset\right\} \\
& \varepsilon_{X}(Y)=\cap\left\{Y_{z}, z \in \breve{X}\right\} .
\end{aligned}
$$

Where $X_{z}$ is the set translated at point $z, X_{z}=\{x+z ; x \in$ $X\}$; and $\breve{X}$ is the transposed set of $X, \breve{X}=\{-x, x \in X\}$. Dilation $\delta_{X}(Y)$ is the locus of the possible $z$ positions of the $\breve{X}_{z}$ transposed element when this latter intersects $Y$. Erosion $\varepsilon_{X}(Y)$, on the other hand, is the location of the possible $z$ positions of the $X_{z}$ element when this latter is contained in $Y$ (Serra, 1988).

Consider the simulation of a set $A$ obtained by the union of $N$ objects $P$, these objects can have any random shape. The model is sequential: the first object is placed randomly in $\mathbb{R}^{3}$. The $x$ position of a new object $P$ may be randomly chosen in the dilation set of the aggregate $A$

$$
A=\cup_{i} P_{i}
$$

defined as:

$$
X_{P}=F\left(\delta_{P}(A)\right)
$$

$F(X)$ is the set of points at the border of $X, F(X)=$ $\left\{z: B_{z} \cap \breve{X} \neq \emptyset\right\}$, with $B$ unit ball.

In the case of a repulsion distance $r$ between objects, the set $X_{P}$ becomes

$$
X_{P \delta B(r)}=F\left(\delta_{P \delta B(r)}(A)\right)
$$


with $B(r)$ sphere of radius $r$. In this way, the new objects will be in contact with or close to the ones already placed. However, the set of objects formed sequentially can easily form cavities not accessible to new objects.

The final compactness of the aggregate can be tuned by placing the objects either in the concavities, or randomly on the aggregate dilation (see Eq.(12)).

Concave points can be extracted by means of closing operator $\varphi$, that can be expressed by dilation and erosion (Serra, 1988)

$$
\varphi_{r}(Y)=\varepsilon_{r}\left(\delta_{r}(Y)\right)
$$

with $r$ the sphere radius. The greater the value of $r$, the larger the size of the concavities. When $r$ tends to infinity, the closure defines the convex envelope, i.e. the totality of the possible convex points. The set $X_{P}^{C V}$ denoting locations of concave points is given by (Fig.3):

$$
X_{P}^{C V}=F\left(\delta_{P}(A)\right) \cap F\left(\varphi_{r}\left(\delta_{P}(A)\right) \backslash \delta_{P}(A)\right)
$$

where $F\left(\varphi_{r}\left(\delta_{P}(A)\right) \backslash \delta_{P}(A)\right)$ denotes the difference between the two sets. Consider $p$ the value of a random variable according to a uniform law on $[0,1]$ and $\alpha \in$ $[0,1]$. If $p \geq \alpha$, the position $x$ of a new object $P$ is chosen randomly in the set $X_{P}$, otherwise the position is chosen in the set $X_{P}^{C V}$.

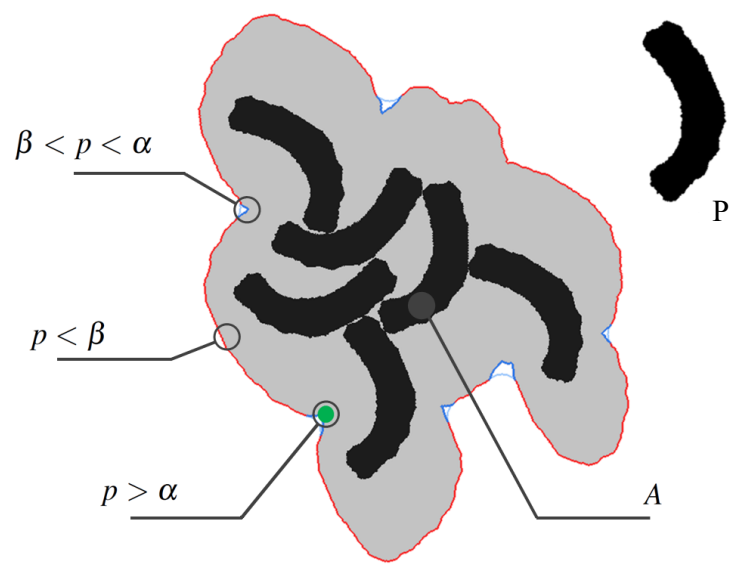

Fig. 3. Illustration during the simulation process for a two-dimensional domain. In the right corner, next object $P$ to be implanted. In black, set $A$ of already located objects. In gray, dilated zone $\delta_{P}(A)$. In red, set $X_{P}$ of admissible positions for $P$. In blue and green, sets of concave points $X_{P}^{C V}$ and $X_{P}^{M}$ respectively.

The $\alpha$ variable tunes the compactness of the set $A$. Therefore, the model can generate an aggregate with few cavities, but the obtained aggregates can still easily be organized with a more or less elongated shape. In order to build even more compact aggregates, one can consider the concavity closest to the center of mass of the set of objects already placed. A new set $X_{P}^{M}$ is defined as:

$$
X_{P}^{M}=x \in X_{P}^{C V} \mid \inf d(x, M)
$$

where $M$ is the center of mass of $A$ and $d(x, M)$ is the euclidean distance between a point $x$ and the point $M$. An illustration of the different sets is proposed in Fig.3, for 2D case.

The parameters $\beta$ and $\alpha$ are used to control the compactness of the final aggregate. It is possible to set $\beta$ as a function of $\alpha$ to reduce the number of parameters. Let $p$ be a uniform random variable on [0;1], $\beta \in[0,1], \alpha \in[0,1]$ and $\alpha \geq \beta$. The position of a new object $P$ is randomly drawn in the set $X_{P}^{M}, X_{P}^{C V}$, or $X_{P}$ depending whether $p \leq \beta, \beta<p \leq \alpha$ or $p>\alpha$ respectively. In particular we can distinguish different cases:

- for $\alpha=\beta=0$ the object $P$ is located randomly on $X_{P}$, producing aggregates with low compactness;

- for $0<\alpha \leq 1, \beta=0$ the object $P$ is located randomly on $X_{P}$ and $X_{P}^{M}$, producing aggregates with low-medium compactness;

- for $\alpha=1, \beta=0$ the object $P$ is located randomly on $X_{P}^{C V}$;

- for $0<\alpha \leq 1,0<\beta<1$ the object $P$ is located either randomly on the aggregate dilation $X_{P}$ either on a concave point of the dilation $X_{P}^{C V}$ or on the concave point closest the the center of mass $X_{P}^{M}$. If $\alpha=\beta, P$ is located either on $X_{P}$ or on $X_{P}^{M}$, producing aggregates with medium-high compactness;

- for $\alpha=\beta=1$ the object $P$ is located on $X_{P}^{M}$, producing aggregated with high compactness.

\section{Size of aggregate}

The size of the final aggregate depends on the one hand on the number of elementary objects and on the other hand on $\alpha$ and $\beta$ compactness parameters. Eq. (7),(8) enables to calculate a size parameter such as gyration radius as a function of the fractal dimension in the case of spherical elementary particles. The morphological model of aggregation can be used with all kinds of object geometries in order to be able to represent various colloidal systems (for example silica or boehmite). Fig. 4 shows realizations of aggregates of rectangular parallelepiped objects modeling alumina nano-platelets derived from Wang (2015), by varying the compactness parameters keeping constant the number of simulated objects. 


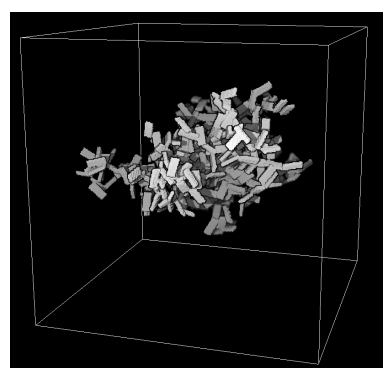

(a)

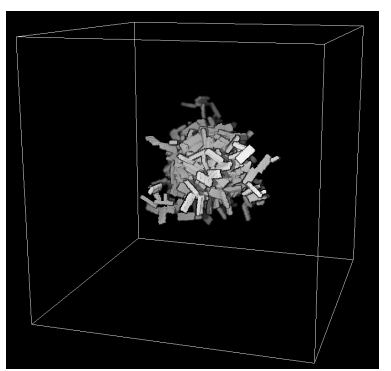

(b)
Fig. 4. Simulation of aggregates of 500 platelets of size $20 \times 8 \times 3$ voxels. Compactness parameters $\beta=0.05$ or $\beta=0.45$ for $(a)$ and $(b)$ respectively. Parameter $\alpha=2 \beta$. Volume size domain is $500^{3}$ voxels. Simulations are performed and rendered using plug im! (plugim, 2018).

Acceleration scheme for spheres with constant radius The simulation of an aggregate of spheres of constant radius is a special case where a very efficient construction algorithm can be defined. A similar scheme is possible for the case of a disc of constant radius. A discrete orthonormal grid in $\mathbb{R}^{3}$ and a volume of simulation of finite size is considered. The Euclidean distance to a set $Y$ from the position $z$ is noted $d(z, Y)$, which is the smallest distance from the point $z$ to a point of the set $Y$. In this discrete space, the morphological dilation of a set $Y$ by a sphere of radius $r$ can be written using a distance function:

$$
\delta_{r}(Y)=Y \cup\{z \in \breve{Y} \mid d(z, Y)<r\}
$$

Smallest possible concavities are considered, i.e. those obtained by a unit size closing. To construct the sets $X_{P}, X_{P}^{C V}$ and $X_{P}^{M}$, it is important not to browse through the entire volume to update the sets each time a new sphere is implemented. Consider a new sphere of radius $r$ to be located at point $x_{C}$. It affects the considered sets only in a cube of side $4 r$ centered in $x_{c}$. Each point of this cube can be assigned according to its previous assignment. The sets $X_{P}$ and $X_{P}^{C V}$ can be updated as follows:

- $\quad$ if $d\left(x, x_{C}\right) \leq r$, then $x \in A$,

- if $r<d\left(x, x_{C}\right) \leq 2 r$ and previously $x \notin A$, then $x \in \delta_{r}(A) / A$,

- $\quad$ if $d\left(x, x_{C}\right)=2 r$ and previously $x \notin \delta_{r}(A)$, then $x \in X_{P}$,

- $\quad$ if $d\left(x, x_{C}\right)=2 r$ and previously $x \in X_{P}$, then $x \in$ $X_{P}^{C V}$.

The center of inertia $M$ is revised with the set $A_{i}$. The updating of the set $X_{P}^{M}$ is then performed by traversing the points of the set $X_{P}^{C V}$

$$
X_{P}^{M}=x \in X_{P}^{C V} \mid \inf d(x, M) .
$$

The storage of the points of each set can be carried out with the help of contiguous arrays allowing to efficiently draw a random position $x$ :

$$
x=l_{X}[p . L]
$$

with $p$ a uniform random variable on $[0,1]$ and $L$ the number of elements of the array $l_{X}$ listing the points of a set $X$. The proposed accelerated algorithm is detailed in Appendix A.

\section{Simulation of large volumes}

In order to use morphological operators, the algorithm works in discrete space with integer numbers for positions and particle shape. This requires objects of a sufficiently large size to achieve the desired precision. The construction of aggregates, even large ones, is extremely fast in the case of disks or spheres with constant radius. For this reason there is an interest in using this model to simulate large volumes of porous structures. Examples of simulations with constant radius of 200000 disks (simulated domain $10000^{2}$ pixels) and 50000 spheres (simulated domain $1000^{3}$ voxels) obtained for calculation times of $4 \mathrm{~s}$ and 40 s respectively (CPU core i7-8850H, 16 Go RAM) are presented Fig.5. The time complexity of the fast scheme is linear in the number of objects to simulate. The algorithms presented in this paper are available under plug im! platform (plugim, 2018).

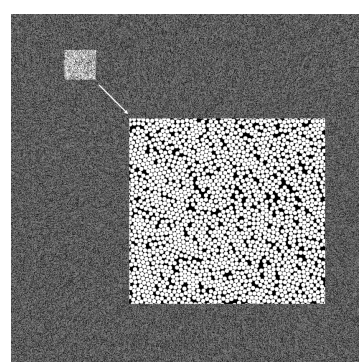

(a)

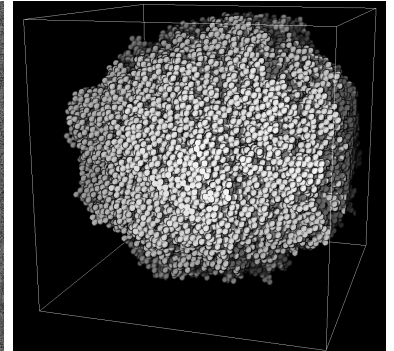

(b)
Fig. 5. Illustration of simulations using the fast scheme $(\beta=0.45, \alpha=2 \beta)$. (a) simulation of 200000 disks of radius 10 pixels, image size is $10000^{2}$. (b) simulation of 50000 spheres of radius 10, volume size is $1000^{3}$. Simulations are performed and rendered using plug im! (plugim, 2018).

\section{CONTROLLED STRUCTURAL PARAMETERS: COMPARISON WITH DLVO SIMULATION}

The geometrical model has been applied for different particles numbers $N$ and different values of compactness factors $\alpha$ and $\beta$. The graphs in Fig.6 show the dependence of the fractal dimension 
of aggregates on the compactness parameters, for different numbers of primary spheres $N . R_{g y r}$ and $d_{f}$ have been calculated using Eq.(9), (8) and (7). The fractal dimension can be linked to a notion of elongation of the aggregate: a fractal dimension close to 2 or 3 corresponds to an aggregate rather elongated shape, rather spherical, respectively. The average $d_{f}$ corresponding to a couple of values for $\alpha$ and $\beta$ and its relative confidence interval are calculated on 1000 aggregates.

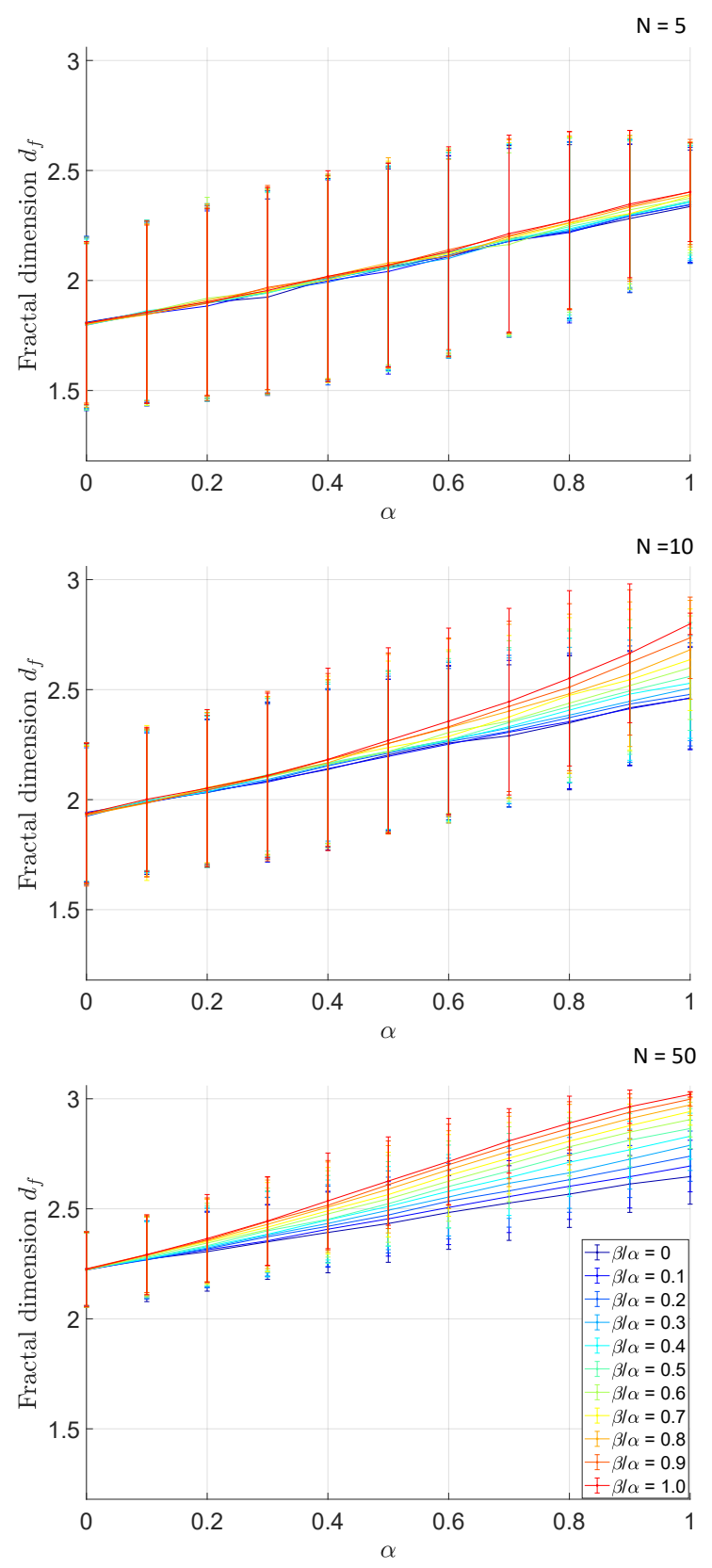

Fig. 6. Fractal dimension of the aggregates generated with the morphological model for different $\alpha$ and $\beta / \alpha$ for $N=5,10,50$. Results are presented as mean values of $d_{f}$ and the relative 95\% confidence intervals on 1000 aggregates.
The relatively wide confidence intervals observed allow simulations that take into account the intrinsic variations of a real system.

The entire range of fractal dimension values obtainable with the model is shown in yellow on the graph in Fig.2. For each $N$ this range has been defined by the extreme values of the confidence intervals amongst all the ones attainable for any combination of $(\alpha, \beta)$. These results show that the morphological aggregation model can reproduce the same aggregates obtained with Brownian dynamics, i.e with fractal dimensions between 1.2 and 3, for aggregates constituted from 2 to unlimited number of particles.

The parametrization of the morphological model is achieved using $\alpha, \beta$ charts to associate all pairs of $d_{f}$, $N$ to the $\alpha, \beta$ couples. In order to draw the $\alpha, \beta$ chart for a given $N$, the average results presented in Fig.6 has to be fit with a two-variable function. This can be easily done with a third degree polynomial:

$$
\begin{aligned}
d_{f}= & p_{00}+p_{10} \cdot x+p_{01} \cdot y+p_{20} \cdot x^{2}+p_{11} \cdot x \cdot y+ \\
& p_{02} \cdot y^{2}+p_{30} \cdot x^{3}+p_{21} \cdot x^{2} \cdot y+ \\
& p_{12} \cdot x \cdot y^{2}+p_{22} \cdot x^{2} \cdot y^{2}+p_{03} \cdot y^{3}
\end{aligned}
$$

where $x=\alpha, y=\beta / \alpha$ and all the coefficient $p_{i, j}$ are a function of $N$. In order to assign all the $\alpha$ and $\beta / \alpha$ couples to an average fractal dimension at a fixed $N$, Eq.(23) must be inverted. The detailed calculations are in Appendix B.

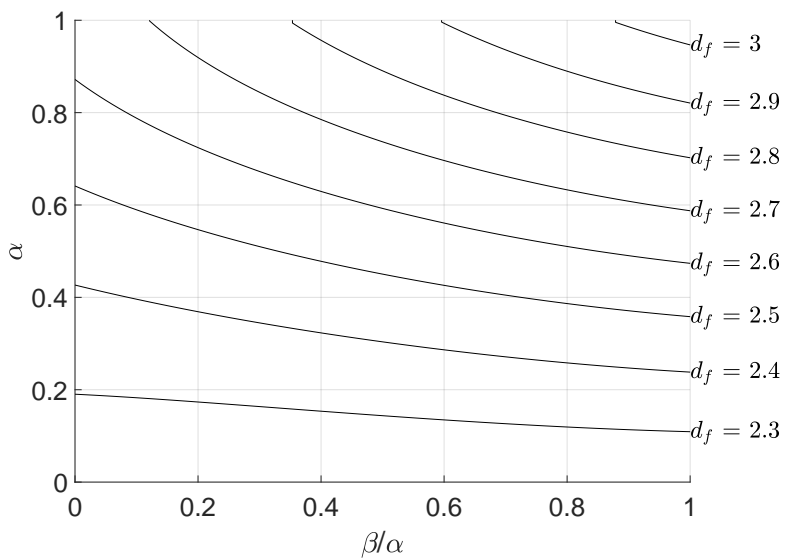

Fig. 7. Example of $\alpha$ and $\beta / \alpha$ chart for aggregates of 50 elementary spheres.

The resolution of the inverted Eq.(23) leads to an $\alpha, \beta$ chart like the one represented in Fig.7 for $N=50$. From now, it is possible to use structural information from Brownian Dynamics i.e. fractal dimension $d_{f}$ and mass $N$ to constrain the morphological model. The values within this range can be obtained by tuning the compactness of the 
aggregates with the two compactness parameters $\alpha$ and $\beta$. In this way, Brownian dynamics simulations acts as a bridge between physical-chemical parameters and the three cluster model parameters $\alpha, \beta$ and $N$. At present, the morphological model builds single aggregates, replicating the Brownian Dynamics aggregates successfully. In a short term, we will directly implement the mass distribution and fractal dimension function $d_{f}(N)$ within the model, in order to mimic the final arrangement of Brownian Dynamics on a larger volume.

\section{RELATIONSHIP BETWEEN THE COMPACTNESS PARAMETERS AND THE PACKING POROSITY}

As explained previously, the interest of the proposed morphological model compared to DLVO simulations is to be able to simulate large systems, representative of porous materials. Once the system is generated, it is possible to calculate textural properties, nitrogen physisorption isotherm (Wang, 2015) or SAXS curves (Sorbier, 2019). However, the present state of the model enables only a sensitivity study of the packing porosity with respect to the compactness parameters $\alpha$ and $\beta$.

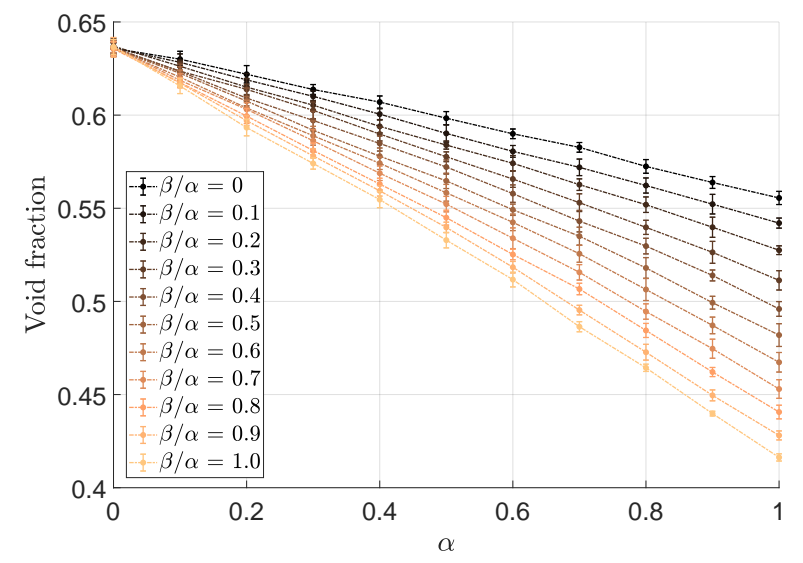

Fig. 8. Void fraction calculated on a 300 pixels control volume filled with identical spheres of radius 10 pixels, for varying $\alpha$ and $\beta / \alpha$. Average value and $95 \%$ confidence interval are calculated on 10 simulations.

Indeed, it is easy to create a single aggregate of spheres with a high $N$ and evaluate the void fraction of a cube contained within it. Compactness parameters are then able to modify the porosity of the structure. Fig. 8 shows the void fraction calculated inside a 300 -pixel length cube completely filled with identical spheres with a radius of 10 pixels. The results are given in terms of the average void fraction and its 95\% confidence interval. The void fraction can vary from 0.41 to 0.63 . However, in order to represent the real $\gamma$ alumina porous structure (that can reach a void fraction up to 0.7 (Kolitcheff, 2017)) it will be necessary to introduce the multi-scale within the algorithm of aggregate generation. This aspect is discussed in the next section.

\section{FRACTAL DIMENSION WITH ANISOTROPIC ELEMENTARY PARTICLES}

The morphological aggregation model has been characterized in terms of $d_{f}(\alpha, \beta, N)$ for isotropic spherical elementary particles. It can also be used to mimic boehmite structure, with aggregates formed by anisotropic platelets (Moreaud, 2012).

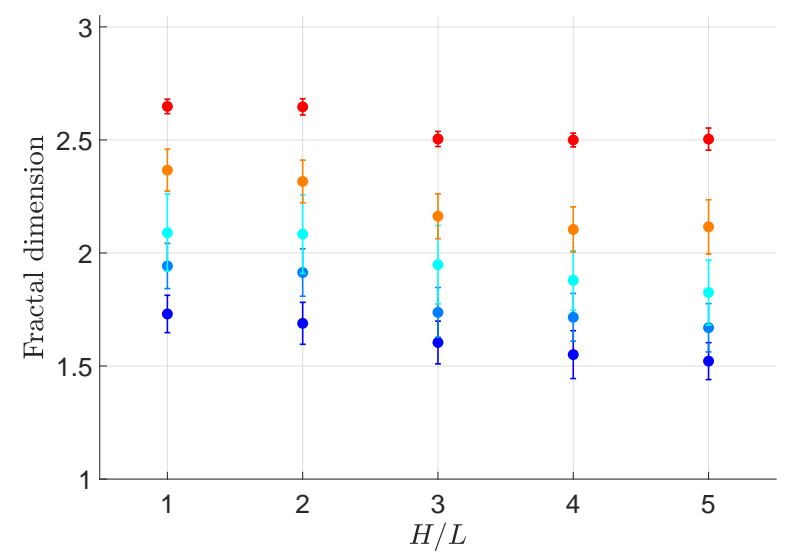

(a)

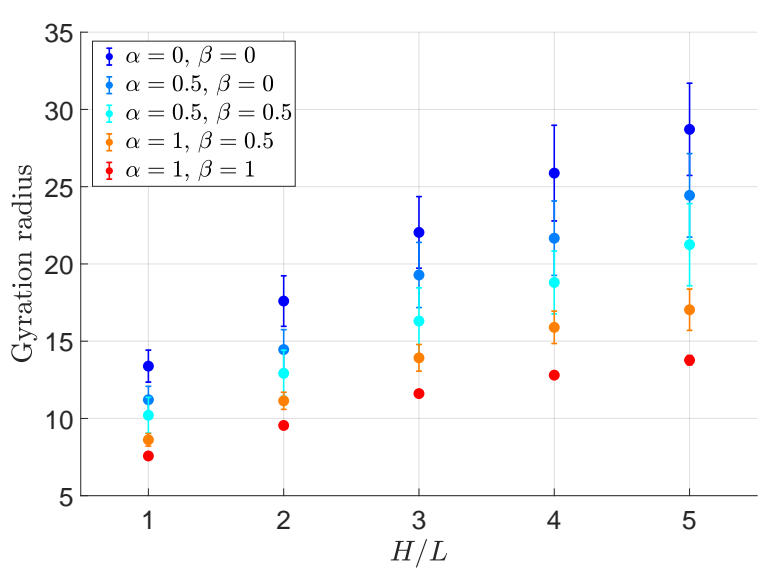

(b)

Fig. 9. Fractal dimension and gyration radius of aggregates of 20 anisotropic platelets with fixed length $L$ (5 pix) and thickness e (3 pix), varying height $H$. The results are presented in terms of average fractal dimension and the $95 \%$ confidence intervals obtained on 40 simulations. The color code of the dots is the same for both graphs.

The fractal dimension is computed with Eq.(7) where $k_{f}=1.2$. The value of $k_{f}$ only shifts $d_{f}(H / L)$ considering the vertical axis and has no impact on its trend. Since $a$ is proportional to the aggregate mass (Gmachowski, 2003), $a$ is calculated as the radius of 
the equivalent volume sphere of the platelet. Fig.9 reports the fractal dimension $d_{f}$ and the gyration radius for different aspect ratios $(H / L)$ of the platelets. For different sets of compactness parameters $\alpha$ and $\beta$ as the aspect ratio of the particles increases, the gyration radius increases significantly, leading to a slight decrease of fractal dimension. Images of such aggregates are presented in Fig.10.

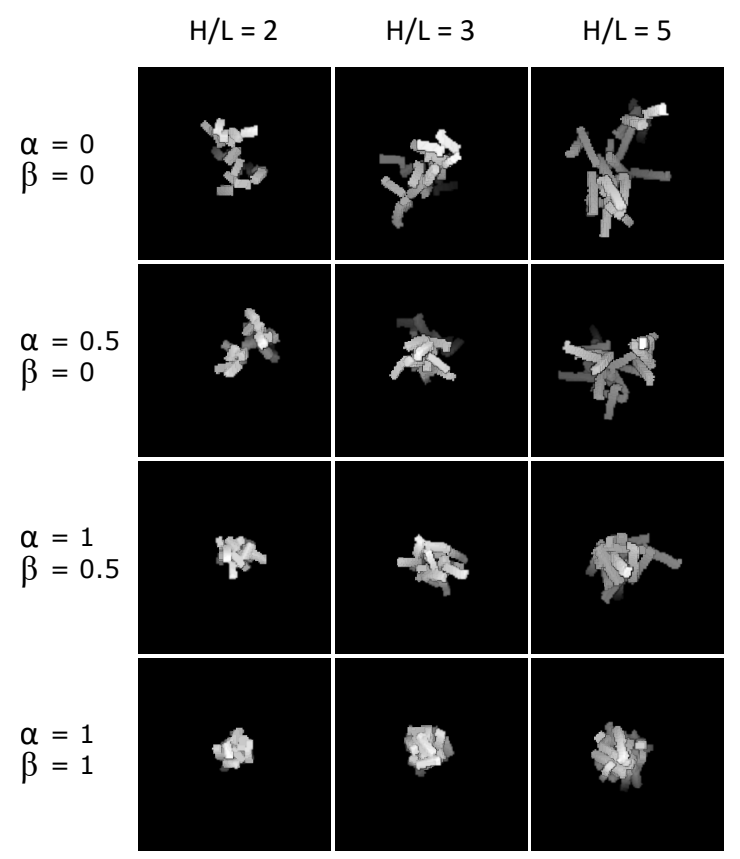

Fig. 10. Aggregates generated using platelets with different shape ratio $H / L$ and different $\alpha$ and $\beta$.

\section{DISCUSSIONS}

Brownian dynamics is often used to investigate the effect of chemical parameters on colloidal aggregation kinetics. The computational time is, however, prohibitive for the simulation of large systems to represent real solid properties. For this reason an easily implementable new random morphological model was developed. As this model is purely mathematical, it is necessary to use the physical information of Brownian dynamics to parameterize the morphological aggregation model in order to build a physically plausible numerical solid. The model can build aggregates composed of any number of elementary objects as we can see in Fig.11. The figure shows the aggregates obtained for different $\alpha$ and $\beta$ values (in which $\beta=0.5 \cdot \alpha$ ) and for a number of elementary objects in the range from 10 to 10000 . It is possible to observe that for higher values of the compactness parameters we obtain more compact structures, similar to spheres, to which corresponds a fractal dimension close to 3. In addition, the compactness of the aggregates also increases with $N$. This is due to the type of growth mechanism, which is particle-cluster, indeed at each iteration an elementary particle is added to the aggregate. This naturally leads to more compact structures than a cluster-cluster mechanism (Schaefer, 1990). This certainly constitutes a limit in representing the porous structures of the alumina range, for which it is possible to measure high porosity (Kolitcheff, 2017). The next essential step will certainly be to introduce the multi-scale within the model. In practical terms, it will be needed to fix a primary fractal dimension $\left(d_{f 1}\right)$ and a primary mass $\left(N_{1}\right)$ distribution in order to build primary aggregates.

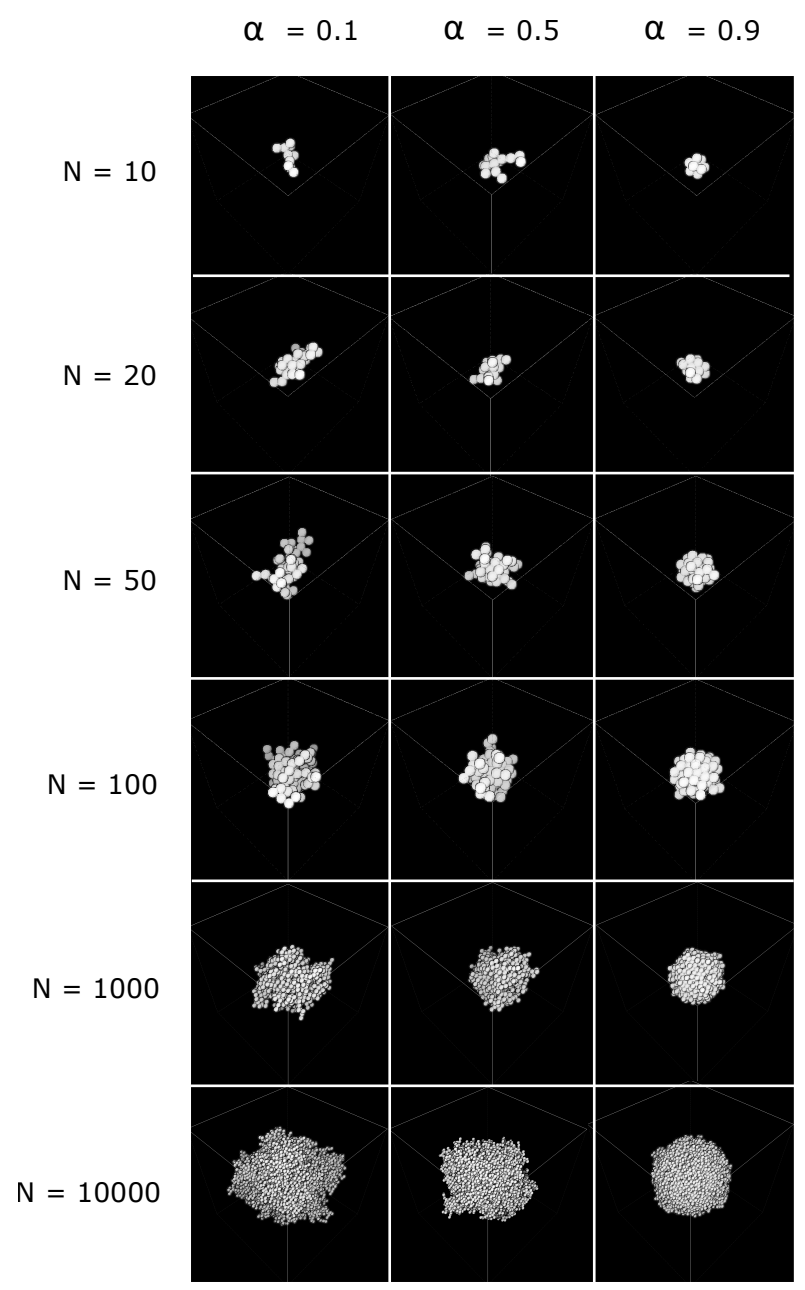

Fig. 11. Aggregates of spheres for different $\alpha, \beta=$ $0.5 \cdot \alpha$

Afterwards, these aggregates will be assembled according to a secondary fractal dimension $\left(d_{f 2}\right)$ and a secondary mass distribution $\left(N_{2}\right) . d_{f 1}, d_{f 2}, N_{1}$ and $N_{2}$ will be chosen according to experimental observation and physical simulation. A third parameter will be required to adjust the probability that an aggregate of mass $i$ is assembled to another aggregate of mass $j$. To define this parameter, the results of a physical model is again essential. 


\section{CONCLUSION}

The objective of this work is to study the effect of aggregation parameters on the final structure of an alumina catalytic support formed within a colloidal suspension under Brownian motion. A new morphological aggregation model is used to simulate single aggregates with a given fractal dimension and number of elementary objects, using Lagrangian simulations result as a reference system. By tuning two compactness parameters and the number of primary objects, all the fractal dimensions observed with Brownian Dynamics can be attained. In the future, this model will be upgraded to receive a mass distribution and its relative fractal dimension function, in order to simulate the final arrangement of Brownian Dynamics on a larger volume. The final numerical solid could be used to perform sensitivity analyses. It will be possible, for example, to study the effect of the aggregation parameters on the textural properties and to understand the impact on mass and heat transport within the porous catalyst carrier.

\section{REFERENCES}

Adamczyk Z, Siwek B, Zembala M, Weroński $\mathrm{P}(1997)$.Influence of Polydispersity on Random Sequential Adsorption of Spherical Particles. J Colloid Interf Sci 185:236-44.

Altendorf H, Jeulin D (2011).Random-walk-based stochastic modeling of three-dimensional fiber systems. Phys Rev E 83:041804.

Anovitz LM, Zhang X, Soltis J, Nakouzi E, Krzysko AJ, Chun J, Schenter GK, Graham TR, Rosso KM, De Yoreo JJ, Stack AG, Bleuel M, Gagnon C, Mildner DFR, Ilavsky J, Kuzmenko I (2018). Effects of Ionic Strength, Salt, and $\mathrm{pH}$ on Aggregation of Boehmite Nanocrystals: Tumbler Small-Angle Neutron and X-ray Scattering and Imaging Analysis. Langmuir 34: 15839-53.

Baranau V, Tallarek U (2014).Random-close packing limits for monodisperse and plydisperse hard spheres. Soft Matter 10:3826.

Cerbelaud M, Ferrando R, Videcoq A (2010). Simulations of heteroaggregation in a suspension of alumina and silica particles: Effect of dilution. $\mathbf{J}$ Chem Phys 132.

Chiche D, Digne M, Revel R, Chanéac C, Jolivet J-P (2008).Accurate Determination of Oxide Nanoparticle Size and Shape Based on XRay Powder Pattern Simulation: Application to Boehmite AlOOH. J Phys Chem-US C 112: 852433.
Cordelair J, Greil P (2004). Flocculation and coagulation kinetics of Al2O3 suspensions. J Eur Ceram Soc 24:2717-22.

Delarue, A. and Jeulin, D. (2001). Multi-scale simulation os spherical aggregates. Image Anal Stereol 20:181-6.

Donev A, Torquato S, Stillinger FH (2005). Neighbor list collision-driven molecular dynamics simulation for nonspherical hard particles. I. Algorithmic details. J Comput Phys 202:737-64.

Długosz M, Zieliński P, Trylska J (2011). Brownian dynamics simulations on CPU and GPU with BD_BOX. J Comput Chem 32:2734-44.

Euzen P, Raybaud P, Krokidis X, Toulhoat H, Le Loarer J-L, Jolivet J-P, Froidefond C (2002). Alumina. In Handbook of Porous Solids. Wiley.

Fabbri R, Da F, Costa L, Torelli JC, Bruno OM (2008). 2D Euclidean distance transform algorithms: A comparative survey. ACM Comput Surv 40.

Filippov AV, Zurita M, Rosner DE (2000). Fractallike Aggregates: Relation between Morphology and Physical. J Colloid Interf Sci 229: 261-73.

Gmachowski L (2003). Mass-radius relation for fractal aggregates of polydisperse particles. Colloid Surface A 224: 45-52.

Greco A, Jeulin D, Serra J (1979). The use of the texture analyser to study sinter structure: application to the morphology of calcium ferrites encountered in basic sinters of rich iron ores. $\mathbf{J}$ Microsc 116: 199-211.

Hamaker (1937). The London-Vander Waals attraction between spherical particles. Physica IV 10:105872.

Hutter (2000). Local Structure Evolution in Particle Network Formation Studied by Brownian Dynamics Simulation. J Colloid Interf Sci 231:337-50.

Iler RK (2004).The chemistry of silica: Solubility, polymerization, colloid and surface properties, and biochemistry. Wiley.

Jeulin D (2000). Random texture models for material structures. Stat Comput 10:121-32.

Jeulin D (2012). Morphology and effective properties of multi-scale random sets:a review. Cr Mecanique 340:219-29.

Jeulin D (2019). Some dense random packings generated by the dead leaves model. Image Anal Stereol 38:3-13.

Krinninger P, Fortini A, Schmidt M (2016). Minimal Model for Dynamic Bonding in Colloidal Transient Networks. Phys Rev E 93. 
Kolitcheff S, Jolimaitre E, Hugon A, Verstraete J, Carrette P-L, Tayakout-Fayolle M (2017). Tortuosity of mesoporous alumina catalyst supports: Influence of the pore network organization. Micropor Mesopor Mat 248:918.

Köylü ÜÖ, Faeth GM, Farias TL, Carvalho MG (1995). Fractal and projected Structure Properties of Soot Aggregates. Combust Flame 100:621-33.

Laganapan AM, Mouas M, Videcoq A, Cerbelaud M, Bienia M, Bowen P, Ferrando R (2015). How colloid-colloid interactions and hydrodynamic effects influence the percolation threshold: A simulation study in alumina suspensions. J Colloid Interf Sci 458: 241-46.

Langevin P (1908) Sur la théorie du mouvement brownien. Cr Acad Sci 17:499-503.

Lebdioua K, Cerbelaud M, Aimable A, Videcoq A (2020) Aggregation behavior of one-patch inverse patchy particles: an experimental and numerical study. hal-02613670.

Lu PJ, Zaccarelli E, Ciulla F, Schofield AB, Sciortino F, Weitz DA (2008) Gelation of particles with short-range attraction. Nature 453: 499-503.

Lubachevsky BD, Stillinger FH, (1990) Geometric properties of random disk packings. J Stat Phys 60:561-83.

Matern, B (2013). Spatial Variation. Lect Notes Stat.

Matheron, G (1975). Random Sets and Integral Geometry. New York. Wiley.

Meakin P (1983). Formation of Fractal Clusters and Networks by Irreversible Diffusion-Limited Aggregation. Phys Rev Lett 51: 1119-22.

Mościński J, Bargieł M, Rycerz Z A, Jacobs P W $M$ (1989). The Force-Biased Algorithm for the Irregular Close Packing of Equal Hard Spheres. Mol Simulat 3:201-12.

Moreaud M, Jeulin D, Morard V, Revel R (2012). TEM image analysis and modelling: Application to boehmite nanoparticles. J Microsc 245:186-99.

Moreaud M, Chaniot J, Fournel T, Becker JM, Sorbier L (2012). Multi-scale stochastic morphological models for 3D complex microstructures. IEEE Worksh Inf Opt Proceedings.

Munhoz AH, de Paiva H, Figueiredo de Miranda L, de Oliveira EC, Andrades RC, Ribeiro RR (2014). Study of Gamma Alumina Synthesis - Analysis of the Specific Surface Area. Adv Sci Tech 87:54-60

Nikunen P, Vattulainen I, Karttunen M (2007). Reptational dynamics in dissipative particle dynamics simulations of polymer melts. Phys Rev E 75 .
Park JD, Myung JS, Ahn KH, (2016). A review on particle dynamics simulation techniques for colloidal dispersions: Methods and applications. Korean J Chem Eng 33:3069-78.

"plug im!" an open access and customizable software for signal and image processing (2018). https://www.plugim.fr.

Sasanuma Y, Kitano Y, Ishitani K (1989). Characterization of industrial materials by small angle X-ray scattering. J Mater Sci 24: 1133-39.

Schaefer DW, Hurd AJ (1990). Growth and Structure of Combustion Aerosols: Fumed Silica. Aerosol Sci Tech 12:876-90.

Schmidt PW, Dacai X (1986). Calculation of the small-angle $\mathrm{X}$-ray and neutron scattering from nonrandom (regular) fractals. Phys Rev A 33: 560.

Schmidt PW (1991). Small-angle scattering studies of disordered, porous and fractal systems. J Appl Crystallogr 24: 414-35.

Serra J (1988). Image analysis and mathematical morphology. Cambridge. Academic Press

Seyed-Ahmadi A, Wachs A (2019). Dynamics and wakes of freely settling and rising cubes. Phys Rev Fluids 4:074304.

Sorbier L, Moreaud M,Humbert S (2019). Small-angle $\mathrm{X}$-ray scattering intensity of multiscale models of spheres. J Appl Crystallogr 52:1348-57.

Sorensen CM (2001). Light Scattering by Fractal Aggregates: A Review. Aerosol Sci Tech 35:64887.

Sorensen CM, Roberts GC (1997). The prefactor of Fractal Aggregates. J Colloid Interf Sci 186:44752.

Tence M, Chevalier JP, Jullien R (1986). On the measurement of the fractal dimension of aggregated particles by electron microscopy: Experimental method, corrections and comparison with numerical models. J Physique 47:1989-98.

Tomchuk OV, Avdeev MV, Bulavin LA (2020). Modeling fractal aggregates of polydisperse particles with tunable dimension. Colloid Surface A 605: 125331.

Torquato S, Jiao Y (2010). Robust algorithm to generate a diverse class of dense disordered and ordered sphere packings via linear programming. Phys Rev E 82:061302

Thouy R, Jullien R (1996) A cluster-cluster aggregation model with tunable fractal dimension. J Phys A-Math Gen 27: 2953.

Verwey EJ, Overbeek JTG. (1948).Theory of the stability of lyophobic colloids: the interaction 
of sol particles having an electric double layer. Elsevier.

Wang H, Pietrasanta A, Jeulin D, Willot F, Faessel M, Sorbier L, Moreaud M (2015).Modelling mesoporous alumina microstructure with 3D random models of platelets. J Microsc 260:287301.

Widom B (1996).Random sequential addition of hard spheres to a volume. J Chem Phys 44:3888.

Witten TA, Sander LM (1983). Diffusion-limited aggregation. Phys Rev B 27:5686.

Zaccarelli E (2007). Colloidal gels: Equilibrium and non-equilibrium routes. J Phys Condens Matter 19.

Zhang G, Torquato S. (2013). Precise algorithm to generate random sequential addition of hard hyperspheres at saturation. Phys Rev E 88:053312. 
APPENDIX A:

SIMULATION ALGORITHM OF A CLUSTER WITH CONTROLLED COMPACTNESS OF IDENTICAL SPHERES

Here is detailed the simulation algorithm of a cluster with controlled compactness of identical spheres.

$X \leftarrow x$ : add point $x$ inside positions table $X, X \rightarrow x$ : delete point $x$ from positions table $X$.

\section{Parameters:}

$N$ : number of spheres;

$\alpha$ : compactness parameter (between 0 and 1 ).

\section{Intialization:}

$\beta=f(\alpha)$;

$x_{C}$ a random position, $X_{A_{i}^{\prime}}=x c, X_{A_{i}^{\prime}}^{C V}=\emptyset, X_{A_{i}^{\prime}}^{M}=\emptyset$, $i=0$.

Iterations: while $i<N$

\{

$p=\cup[0 ; 1]$

if $(p<\alpha) x_{C}=$ random position in $X_{A_{i}^{\prime}}^{C V}$;

if $(p<\beta) x_{C}=$ random position in $X_{A_{i}^{\prime}}^{M}$;

if ( $x_{c}$ is empty) $x_{C}=$ random position in $X_{A_{i}^{\prime}}$;

for each position $x$ in a cube of size $4 r$ centered in $x_{C}$

\{

if $\left(d\left(x, x_{C}\right) \leq r\right) A_{i} \leftarrow x$;

if $\left(d\left(x, x_{C}\right) \leq 2 r\right) X_{A_{i}^{\prime}} \rightarrow x, X_{A_{i}^{\prime}}^{C V} \rightarrow x$;

if $\left(d\left(x, x_{C}\right)=2 r\right)$

\{

if $\left(x \notin X_{A_{i}^{\prime}}\right) X_{A_{i}^{\prime}} \leftarrow x$;

\}

if $\left(x \in X_{A_{i}^{\prime}}\right) X_{A_{i}^{\prime}}^{C V} \leftarrow x, X_{A_{i}^{\prime}} \rightarrow x$;

\}

Update $M$ with $A_{i}$;

Update $X_{A_{i}^{\prime}}^{M}$ with $X_{A_{i}^{\prime}}^{C V}$ and $M$;

$i=i+1$

\}

\section{APPENDIX B:}

THE CONSTRUCTION OF $\alpha, \beta$ CHARTS WITH THE CLUSTER MODEL

The parametrization of the morphological model is achieved using $\alpha, \beta$ charts to associate all pairs of $d_{f}, N$ to the $\alpha, \beta$ couples. In order to draw these charts, the results of the morphological model are fit with a twovariable $3^{\text {rd }}$ degree function:

$$
\begin{aligned}
d f= & p_{00}+p_{10} \cdot x+p_{01} \cdot y+p_{20} \cdot x^{2}+p_{11} \cdot x \cdot y+ \\
& p_{02} \cdot y^{2}+p_{30} \cdot x^{3}+p_{21} \cdot x^{2} \cdot y+p_{12} \cdot x \cdot y^{2}+ \\
& p_{22} \cdot x^{2} \cdot y^{2}+p_{03} \cdot y^{3}
\end{aligned}
$$

where $x=\alpha, y=\beta / \alpha$ and all the coefficient $p_{i, j}$ are a function of $N$. The Eq.(24) is solved with respect to $\alpha$. The resolution gives the intervals $\beta / \alpha$ that, together with $\alpha$ enables to build aggregates with an average fractal dimension. The zeros of cubic function are to be found in $x$

$$
x^{3}+a_{2} \cdot x^{2}+a_{1} \cdot x+a_{0} \cdot-d f=0
$$

where

$$
\begin{aligned}
& a_{2}=\left(p_{20}+p_{21} \cdot y\right) / p_{30} \\
& a_{1}=\left(p_{10}+p_{11} \cdot y+p_{12} \cdot y^{2}\right) / p_{30} \\
& a_{0}=\left(p_{03} \cdot y^{3}+p_{02} \cdot y^{2}+p_{01} \cdot y+p_{00}-d f\right) / p_{30} .
\end{aligned}
$$

Given

$$
\begin{aligned}
& q=1 / 3 \cdot a_{1}-1 / 9 \cdot a_{2}^{2} \\
& r=1 / 6 \cdot\left(a_{1} \cdot a_{2}-3 \cdot a_{0}\right)-1 / 27 \cdot a_{2}^{3}
\end{aligned}
$$

$s_{1}$ and $s_{2}$ are defined as the real numbers

$$
\begin{aligned}
& s_{1}=\left(r+\left(q^{3}+r^{2}\right)^{1 / 2}\right)^{1 / 3} \\
& s_{2}=\left(r-\left(q^{3}+r^{2}\right)^{1 / 2}\right)^{1 / 3} .
\end{aligned}
$$

For a given value of $y$ only real solutions between 0 and 1 are considered within the three roots of the inverted cubic function:

$$
\begin{aligned}
& x_{1}=\left(s_{1}+s_{2}\right)-a_{2} / 3 \\
& x_{2}=-0.5 \cdot\left(s_{1}+s_{2}\right)-a_{2} / 3+0.5 \cdot i\left(s_{1}+s_{2}\right)^{1 / 3} \\
& x_{3}=-0.5 \cdot\left(s_{1}+s_{2}\right)-a_{2} / 3-0.5 \cdot i\left(s_{1}+s_{2}\right)^{1 / 3} .
\end{aligned}
$$

